\title{
Celebrating Dr Olga Kennard
}

\author{
Suzanna C. Ward \\ The Cambridge Crystallographic Data Centre (CCDC), Cambridge, United Kingdom \\ E-mail:ward@ccdc.cam.ac.uk
}

Olga Kennard is a true pioneer. Following a shared vision with JD Bernal that the collective use of data would lead to new knowledge and generate new insights ${ }^{1}$, Olga Kennard established the Cambridge Structural Database (CSD) ${ }^{2}$ in 1965. Some fifty-five years later she was awarded the twelfth Ewald Prize ${ }^{3}$ for her remarkable contribution to the field of crystallography and the development of crystallographic databases.

Today the CSD contains over 1.1 million crystal structures and every single entry is enriched and annotated by experts at the CCDC to aid the discoverability of data and knowledge from the resource. This rich resource of trusted data is used by researchers and educators worldwide in both academia and industry. It is routinely used to help develop new pharmaceutical drugs and has been utilised in a wide range of applications from structure solution to gas storage and separation. Efforts to create the CSD also led Olga Kennard to help set up the Protein Data Bank (PDB) ${ }^{4}$ and be a guiding influence for the establishment of the Nucleic Acid Sequence Database 5 , after persuading the European Molecular Biology Organisation to embark upon it. Nowadays, to complete this circle, the small molecules contained in biological structures archived in the PDB are validated using CCDC software which incorporates the knowledge embodied in the CSD.

The presentation will celebrate Olga Kennard's inspirational career and how she has changed our world of crystallography and beyond. It will explore her scientific journey from early publications outlining procedures for determining accurate cell dimensions ${ }^{6}$ to the establishment of the CSD and the creation of a modern database with structure-based searching. Most importantly this presentation will thank Olga Kennard for her amazing contribution to science.

1. Bernal's Vision: From Data to Insight - J.D.Bernal Lecture delivered at Birkbeck Kollere, London in 1995 by Dr Olga Kennard

2. Groom, C. R., Bruno, I. J., Lightfoot, M. P. \& Ward, S. C. (2016). Acta Cryst. B72, 171-179, DOI: 10.1107/S2052520616003954

3. https://www.iucr.org/iucr/ewald-prize

4. https://www.wwpdb.org/

5. https://www.ebi.ac.uk/ena

6. O.Weisz, W.Cochran \& W.F.Cole, 1948 Acta Cryst. 1, 83-88

Keywords: Cambridge Structural Database 